\title{
On a doubly degenerate parabolic equation with a nonlinear damping term
}

Wenbin $\mathrm{Xu}^{1 *}$

"Correspondence:

wbxu@jmu.edu.cn

'School of Sciences, Jimei University, Xiamen 361021, Fujian

Province, China

\section{Springer}

\begin{abstract}
Consider a double degenerate parabolic equation arising from the electrorheological fluids theory and many other diffusion problems. Let $v_{\varepsilon}$ be the viscous solution of the equation. By showing that $\left|\nabla v_{\varepsilon}\right| \in L^{\infty}\left(0, T ; L_{\mathrm{loc}}^{p(x)}(\Omega)\right)$ and $\nabla v_{\varepsilon} \rightarrow \nabla v$ almost everywhere, the existence of weak solutions is proved by the viscous solution method. By imposing some restriction on the nonlinear damping terms, the stability of weak solutions is established. The innovation lies in that the homogeneous boundary value condition is substituted by the condition $\left.a(x)\right|_{x \in \partial \Omega}=0$, where $a(x)$ is the diffusion coefficient. The difficulties come from the nonlinearity of $|\nabla v|^{p(x)-2}$ as well as the nonlinearity of $|v|^{\alpha(x)}$.
\end{abstract}

MSC: 35K55; 35K92; 35K85; 35R35

Keywords: Electrorheological fluids theory; Damping term; Stability; Nonlinearity

\section{Introduction}

The initial-boundary value problem of a doubly degenerate parabolic equation

$$
\begin{aligned}
& v_{t}=\operatorname{div}\left(a(x)|v|^{\alpha(x)}|\nabla v|^{p(x)-2} \nabla v\right)+f(x, t, v,|\nabla v|), \quad(x, t) \in Q_{T}=\Omega \times(0, T), \\
& v(x, 0)=v_{0}(x), \quad x \in \Omega, \\
& v(x, t)=0, \quad(x, t) \in \partial \Omega \times(0, T),
\end{aligned}
$$

is considered, where $p(x)>1, \alpha(x)$ and $a(x)$ are nonnegative $C(\bar{\Omega})$ functions, $f(x, t, v,|\nabla v|)$ is a continuous function and is called the nonlinear damping term. This equation comes from non-Newtonian fluid, the so-called electrorheological fluids, the heat conduction, and many other diffusion problems.

What first caught our attention is the heat conduction equation with a damping term

$$
v_{t}=v \Delta v-\gamma|\nabla v|^{2} .
$$

The author of [5] showed that the uniqueness is not true. The author of [31] and [33] generalized the results of [5] to a more general equation

$$
v_{t}=\Delta v-g(x)|v|^{q-1}|\nabla v|^{2},
$$

(c) The Author(s) 2021. This article is licensed under a Creative Commons Attribution 4.0 International License, which permits use, sharing, adaptation, distribution and reproduction in any medium or format, as long as you give appropriate credit to the original author(s) and the source, provide a link to the Creative Commons licence, and indicate if changes were made. The images or other third party material in this article are included in the article's Creative Commons licence, unless indicated otherwise in a credit line to the material. If material is not included in the article's Creative Commons licence and your intended use is not permitted by statutory regulation or exceeds the permitted use, you will need to obtain permission directly from the copyright holder. To view a copy of this licence, visit http://creativecommons.org/licenses/by/4.0/. 
where $q \geq 1, g(x) \geq 0$ and there is a point $x_{0} \in \Omega$ such that $g\left(x_{0}\right)>0$. Based on these facts, one may conjecture that the heat conduction equation with a nonlinear damping term

$$
v_{t}=v \Delta v+f(x, t, v,|\nabla v|)
$$

is ill-posed.

The second aspect that attracted our attention is the so-called electrorheological fluids equation

$$
v_{t}=\operatorname{div}\left(|\nabla v|^{p(x)-2} \nabla v\right)
$$

which has been widely studied by many mathematicians, one can refer to $[8,9,11-16]$ and the references therein. A more complicated equation

$$
v_{t}=\operatorname{div}\left(a(x, t, v)|\nabla v|^{p(x)-2} \nabla v\right)
$$

was studied in $[2,3]$. Though the existence of weak solutions to equation (1.8) has been shown, the uniqueness result only for the case of $|a(x, t, u)-a(x, t, v)| \leq \omega(|u-v|)$,

$$
\lim _{\varepsilon \rightarrow \infty} \int_{\varepsilon}^{1} \frac{d s}{\omega(s)^{\beta}}=\infty
$$

has been proved, where $1<\beta<\frac{p^{+}}{p^{+}-1}$. In other words, the general uniqueness problem of equation (1.8) remains open till today.

Let $a(x)$ satisfy

$$
a(x)=0, \quad x \in \partial \Omega, \quad a(x)>0, \quad x \in \Omega .
$$

Then equation (1.1) is degenerate on the boundary $\partial \Omega$. If $\alpha(x)=0, p(x)=p$ is a constant and $f(x, t, v,|\nabla v|)=0$, on the stability of weak solutions, that the degeneracy of $\left.a(x)\right|_{x \in \partial \Omega}$ may take place of the usual boundary value condition (1.3) was revealed in [20, 21]. Moreover, whether

$$
v_{t}=\operatorname{div}\left(a(x)|v|^{\alpha(x)}|\nabla v|^{p(x)-2} \nabla v\right)+\sum_{i=1}^{N} \frac{\partial b_{i}(v)}{\partial x_{i}}
$$

or

$$
v_{t}=\operatorname{div}\left(a(x)|\nabla v|^{p-2} \nabla v\right)-b(x)|\nabla v|^{q}
$$

similar results have been obtained in [27] and [25] respectively. For the other related papers, one can refer to $[19,23,24]$ etc.

For equation (1.1), compared with equation (1.7), there exists another diffusion coefficient $a(x)$. Compared with equation (1.11), the convective term $\sum_{i=1}^{N} \frac{\partial b_{i}(v)}{\partial x_{i}}$ is replaced by a nonlinear damping term $f(x, t, v,|\nabla v|)$. Considering all these factors, compared the damping term $f(x, t, v,|\nabla v|)$ with the degeneracy of $\left.a(x)\right|_{x \in \partial \Omega}$, the latter plays a leading role when the uniqueness problem is considered. Maybe such a conclusion can be explained by the 
fact that equation (1.1) represents the model that the diffusion process is more dominant than the damping phenomena. For example, for an epidemic model of diseases, it is impossible to know in advance that $v=0$ on the boundary $\partial \Omega$. Thus, imposing the boundary value condition (1.3) seems unreasonable, while the condition $\left.a(x)\right|_{x \in \partial \Omega}=0$ can be explained as some anthropogenic interferences are made to control the epidemic across the border $\partial \Omega$. In accord with this fact, in theory, we conjecture that under the condition $\left.a(x)\right|_{x \in \partial \Omega}=0$, one can deduce that $v=0$ on the boundary $\partial \Omega$. This conjecture was partially proved in [22] several years ago, and we are not ready to discuss this conjecture in this paper for the time being.

The main aim of this paper is to establish the well-posedness theory for equation (1.1). To accomplish this aim, the nonlinearity of $|v|^{\alpha(x)}$ and the nonlinearity of the damping term $f(x, t, v,|\nabla v|)$ are the main difficulties to overcome. The extinction, the positivity, the large time behavior of the solutions and $v=0$ on the boundary $\partial \Omega$, all these important contents remain to be studied in the future.

Let us give the definition of weak solution.

Definition 1.1 If $v(x, t)$ satisfies

$$
v \in L^{\infty}\left(Q_{T}\right), \quad \frac{\partial v}{\partial t} \in \mathbf{W}^{\prime}\left(Q_{T}\right), \quad a(x)|v|^{\alpha(x)}|\nabla v|^{p(x)} \in L^{1}\left(Q_{T}\right)
$$

and for any function $\varphi \in C_{0}^{1}\left(Q_{T}\right)$,

$$
\iint_{Q_{T}}\left(\frac{\partial v}{\partial t} \varphi+a(x)|v|^{\alpha(x)}|\nabla v|^{p(x)-2} \nabla v \cdot \nabla \varphi\right) d x d t=\iint_{Q_{T}} f(x, t, v,|\nabla v|) \varphi d x d t
$$

and

$$
\lim _{t \rightarrow 0} \int_{\Omega} v(x, t) \phi(x) d x=\int_{\Omega} v_{0}(x) \phi(x) d x
$$

for any $\phi(x) \in C_{0}^{\infty}(\Omega)$, then we say that $v(x, t)$ is a weak solution of equation (1.1) with initial value (1.2).

Here, the basic Banach space $\mathbf{W}\left(Q_{T}\right)$ and its dual space $\mathbf{W}^{\prime}\left(Q_{T}\right)$ are defined by Antontsev and Shmarev in [2]. In addition, let

$$
p^{+}=\max _{x \in \bar{\Omega}} p(x), \quad p^{-}=\min _{x \in \bar{\Omega}} p(x)
$$

and set $q(x)=\frac{p(x)}{p(x)-1}$ as usual. The main results in this paper are the following theorems.

Theorem 1.2 If $a(x) \in C(\bar{\Omega})$ satisfies (1.10), $f(x, t, v,|\nabla v|) \leq 0$ when $v<0$,

$$
0 \leq v_{0}(x) \in L^{\infty}(\Omega), \quad a(x)\left|v_{0}\right|^{\alpha(x)}\left|\nabla v_{0}\right|^{p(x)} \in L^{1}(\Omega)
$$

and

(i) when $p^{-} \geq 2$,

$$
|f(x, t, v,|\nabla v|)| \leq c(a(x)|v|)^{\frac{2 \alpha(x)}{p(x)}}|\nabla v|^{2},
$$


(ii) when $p^{-}>1$,

$$
|f(x, t, v,|\nabla v|)| \leq c(a(x)|v|)^{\frac{\alpha(x)}{p(x)}}|\nabla v| .
$$

Then equation (1.1) with initial value (1.2) has a nonnegative solution $v(x, t)$.

Theorem 1.3 Let $u(x, t)$ and $v(x, t)$ be two solutions of equation (1.1) with the initial values $u_{0}(x)$ and $v_{0}(x)$ respectively and with the same homogeneous boundary value condition

$$
u(x, t)=v(x, t)=0, \quad(x, t) \in \partial \Omega \times(0, T) .
$$

If $\alpha(x) \in C_{0}^{1}(\Omega), a(x) \in C(\bar{\Omega})$ satisfy (1.10) and the nonlinear damping term satisfies

(i) when $p^{-} \geq 2$,

$$
\begin{aligned}
& |f(x, t, u,|\nabla u|)-f(x, t, v,|\nabla v|)| \\
& \quad \leq c|u-v|\left[(a(x)|v|)^{\frac{2 \alpha(x)}{p(x)}}|\nabla v|^{2}+(a(x)|u|)^{\frac{2 \alpha(x)}{p(x)}}|\nabla u|^{2}\right],
\end{aligned}
$$

(ii) when $p^{-}>1$,

$$
\begin{aligned}
& |f(x, t, u,|\nabla u|)-f(x, t, v,|\nabla v|)| \\
& \quad \leq c|u-v|\left[(a(x)|v|)^{\frac{\alpha(x)}{p(x)}}|\nabla v|+(a(x)|u|)^{\frac{\alpha(x)}{p(x)}}|\nabla u|\right] .
\end{aligned}
$$

Then

$$
\int_{\Omega}|u(x, t)-v(x, t)| d x \leq c \int_{\Omega}\left|u_{0}(x)-v_{0}(x)\right| d x, \quad \text { a.e. } t \in[0, T) .
$$

In particular, if $\alpha(x) \equiv 0$, besides Theorem 1.3, we have the following theorem.

Theorem 1.4 Let $u(x, t)$ and $v(x, t)$ be two solutions of equation (1.1) with the initial values $u_{0}(x)$ and $v_{0}(x)$ respectively and with the same homogeneous boundary value condition (1.18). If $\alpha(x) \equiv 0, p^{-} \geq 2, a(x) \in C(\bar{\Omega})$ satisfy(1.10) and the nonlinear damping term satisfies

$$
|f(x, t, u,|\nabla u|)-f(x, t, v,|\nabla v|)| \leq c a(x)\left(|\nabla v|^{2}+|\nabla u|^{2}\right)
$$

then

$$
\int_{\Omega}|u(x, t)-v(x, t)|^{2} d x \leq c \int_{\Omega}\left|u_{0}(x)-v_{0}(x)\right|^{2} d x, \quad \text { a.e. } t \in[0, T) .
$$

Moreover, since the diffusion coefficient $a(x)$ satisfies (1.10), we can obtain a stability theorem without the boundary value condition (1.18).

Theorem 1.5 Let $u(x, t)$ and $v(x, t)$ be two solutions of equation (1.2) with the initial values $u_{0}(x)$ and $v_{0}(x)$ respectively. If $\alpha(x) \in C_{0}^{1}(\Omega), a(x)$ satisfies

$$
\lambda^{1-p^{+}} \int_{\Omega \backslash \Omega_{\lambda}}|\nabla a|^{p(x)} d x \leq c
$$


and the nonlinear damping term satisfies (1.16) and (1.19), then the stability of weak solutions is true in the sense of (1.21).

Here and in what follows, $\lambda>0$ is a small enough constant, and we define $\Omega_{\lambda}=\{x \in \Omega$ : $a(x)>\lambda\}$.

Compared with Theorem 1.3, there is not boundary value condition (1.18) in Theorem 1.4. Instead, condition (1.24) is imposed. Comparing with other related works [2, 3], the most distinctive assumption in this paper is that $\alpha(x) \in C_{0}^{1}(\Omega)$. Since

$$
\left|u^{\alpha(x)}-v^{\alpha(x)}\right| \leq c|u-v|^{\alpha(x)}
$$

is always true, and in particular $\inf _{x \in \bar{\Omega}} \alpha(x)=0$, but $\max _{x \in \bar{\Omega}} \alpha(x)$ can be larger than $\frac{p^{+}}{p^{+}-1}$. This fact implies that when $\alpha(x) \in C_{0}^{1}(\Omega), u^{\alpha(x)}$ is beyond the restriction (1.9). So, Theorem 1.3 and Theorem 1.4 have some essential improvements from the works [2,3]. In the next research, we will try to do some work when $\alpha(x)$ is not limited to $C_{0}^{1}(\Omega)$. By the way, from [4,5] [33] and [31], in order to obtain the well-posedness of weak solutions to equation (1.1), the damping term $f(x, t, u, \nabla u)$ must satisfy some restrictions, for example, condition (1.19) and condition (1.20) in our paper. A similar condition was first introduced by Karlsen and Ohlberger in their paper [10], in which the uniqueness of weak solutions to the equation

$$
u_{t}=\nabla(K(x, t) \nabla A(u))+\sum_{i=1}^{N} \frac{\partial b_{i}(u, x, t)}{\partial x_{i}}+c(u, x, t)
$$

is proved. Although, as one of the reviewers pointed out, condition (1.19) or condition (1.20) is reasonable, are there other conditions to replace condition (1.19) or condition (1.20)? This is also an interesting problem.

\section{The proof of Theorem 1.2}

In this section, we prove Theorem 1.2.

Let us consider an approximate problem

$$
\begin{aligned}
& v_{\varepsilon t}-\operatorname{div}\left((a(x)+\varepsilon)\left(\left|v_{\varepsilon}\right|^{\alpha(x)}+\varepsilon\right)\left|\nabla v_{\varepsilon}\right|^{p(x)-2} \nabla v_{\varepsilon}\right)=f\left(x, t, v_{\varepsilon},\left|\nabla v_{\varepsilon}\right|\right), \quad(x, t) \in Q_{T}, \\
& v_{\varepsilon}(x, t)=0, \quad(x, t) \in \partial \Omega \times(0, T), \\
& v_{\varepsilon}(x, 0)=v_{\varepsilon 0}(x), \quad x \in \Omega
\end{aligned}
$$

where $v_{\varepsilon 0} \in C_{0}^{\infty}(\Omega),\left|v_{\varepsilon 0}\right|_{L^{\infty}(\Omega)} \leq\left|v_{0}\right|_{L^{\infty}(\Omega)}, a(x)\left|\nabla v_{\varepsilon 0}\right|^{(x) p}$ converges to $a(x)\left|\nabla v_{0}(x)\right|^{p}$ in $L^{1}(\Omega)$ uniformly. Since $f\left(x, t, v_{\varepsilon},\left|\nabla v_{\varepsilon}\right|\right) \leq 0$ when $v_{\varepsilon}<0$ and satisfies (1.16) or (1.17), the above problem (2.1)-(2.3) has a unique nonnegative solution $v_{\varepsilon} \in L^{\infty}\left(0, T ; W_{\text {loc }}^{1, p}(\Omega)\right)$, and

$$
\left\|v_{\varepsilon}\right\|_{L^{\infty}\left(Q_{T}\right)} \leq c,
$$

one can refer to $[3,7,18]$ for details.

Lemma 2.1 If $u_{\varepsilon} \in L^{\infty}\left(0, T ; L^{2}(\Omega)\right) \cap \mathbf{W}\left(Q_{T}\right),\left\|u_{\varepsilon t}\right\|_{\mathbf{W}^{\prime}\left(Q_{T}\right)} \leq c,\left\|\nabla\left(\left|u_{\varepsilon}\right|^{q-1} u_{\varepsilon}\right)\right\|_{p, Q_{T}} \leq c$, then there is a subsequence of $\left\{u_{\varepsilon}\right\}$ which is relatively compact in $L^{s}\left(Q_{T}\right)$ with $s \in(1, \infty)$. Here, $q \geq 1$. 
This lemma can be found in [17].

Proof of Theorem 1.2 At first, let us multiply (2.1) by $v_{\varepsilon}$. Since $f(x, t, v,|\nabla v|) \leq 0$ when $v<0$ and satisfies (1.16), by the Young inequality, we have:

(i) when $p^{-} \geq 2, f\left(x, t, v_{\varepsilon},\left|\nabla v_{\varepsilon}\right|\right)$ satisfies (1.16), we have

$$
\begin{aligned}
& \frac{1}{2} \int_{\Omega} v_{\varepsilon}^{2} d x+\iint_{Q_{T}}(a(x)+\varepsilon)\left(\left|v_{\varepsilon}\right|^{\alpha(x)}+\varepsilon\right)\left|\nabla v_{\varepsilon}\right|^{p(x)} d x d t \\
& \quad=\frac{1}{2} \int_{\Omega} v_{\varepsilon 0}^{2} d x+\iint_{Q_{T}} f\left(x, t, v_{\varepsilon}, \mid \nabla v_{\varepsilon}\right) \mid v_{\varepsilon} d x d t \\
& \quad \leq \frac{1}{2} \int_{\Omega} v_{\varepsilon 0}^{2} d x+c \iint_{Q_{T}}(a(x)|v|)^{\frac{2 \alpha(x)}{p(x)}}|\nabla v|^{2} d x d t \\
& \quad \leq \frac{1}{2} \int_{\Omega} v_{\varepsilon 0}^{2} d x+\iint_{Q_{T}}\left[\varepsilon a(x)\left|v_{\varepsilon}\right|^{\alpha(x)}\left|\nabla v_{\varepsilon}\right|^{p(x)}+c(\varepsilon)\right] d x d t \\
& \quad \leq c
\end{aligned}
$$

(ii) when $p^{-}>1, f\left(x, t, v_{\varepsilon},\left|\nabla v_{\varepsilon}\right|\right)$ satisfies (1.17), we have

$$
\begin{aligned}
& \frac{1}{2} \int_{\Omega} v_{\varepsilon}^{2} d x+\iint_{Q_{T}}(a(x)+\varepsilon)\left(\left|v_{\varepsilon}\right|^{\alpha(x)}+\varepsilon\right)\left|\nabla v_{\varepsilon}\right|^{p(x)} d x d t \\
& \quad=\frac{1}{2} \int_{\Omega} v_{\varepsilon 0}^{2} d x+\iint_{Q_{T}} f\left(x, t, v_{\varepsilon}, \mid \nabla v_{\varepsilon}\right) \mid v_{\varepsilon} d x d t \\
& \quad \leq \frac{1}{2} \int_{\Omega} v_{\varepsilon 0}^{2} d x+c \iint_{Q_{T}}(a(x)|v|)^{\frac{\alpha(x)}{p(x)}}|\nabla v| d x d t \\
& \quad \leq \frac{1}{2} \int_{\Omega} v_{\varepsilon 0}^{2} d x+\iint_{Q_{T}}\left[\varepsilon a(x)\left|v_{\varepsilon}\right|^{\alpha(x)}\left|\nabla v_{\varepsilon}\right|^{p(x)}+c(\varepsilon)\right] d x d t \\
& \quad \leq c .
\end{aligned}
$$

Then

$$
\iint_{Q_{T}} a(x)\left|v_{\varepsilon}\right|^{\alpha(x)}\left|\nabla v_{\varepsilon}\right|^{p(x)} d x d t \leq \iint_{Q_{T}}(a(x)+\varepsilon)\left(\left|v_{\varepsilon}\right|^{\alpha(x)}+\varepsilon\right)\left|\nabla v_{\varepsilon}\right|^{p(x)} d x d t \leq c,
$$

which implies

$$
\iint_{Q_{T}} a(x)\left|\nabla v_{\varepsilon}^{\frac{\alpha(x)}{p(x)}+1}\right|^{p(x)} d x d t \leq c
$$

Secondly, according to the definition of Banach space $\mathbf{W}\left(Q_{T}\right)$ [2], $C_{0}^{\infty}\left(Q_{T}\right)$ is dense in $\mathbf{W}\left(Q_{T}\right)$. Now, for any $u \in C_{0}^{\infty}\left(Q_{T}\right),\|u\|_{W\left(Q_{T}\right)}=1$, we have

$$
\begin{aligned}
\left\langle v_{\varepsilon}, u\right\rangle= & -\iint_{Q_{T}}(a(x)+\varepsilon)\left(\left|v_{\varepsilon}\right|^{\alpha(x)}+\varepsilon\right)\left|\nabla v_{\varepsilon}\right|^{p(x)-2} \nabla v_{\varepsilon} \cdot \nabla u d x d t \\
& +\iint_{Q_{T}} f\left(x, t, v_{\varepsilon},\left|\nabla v_{\varepsilon}\right|\right) u d x d t
\end{aligned}
$$


According to condition (1.16) or (1.17), by the Young inequality, we easily deduce

$$
\begin{aligned}
& \left|\iint_{Q_{T}} f\left(x, t, v_{\varepsilon},\left|\nabla v_{\varepsilon}\right|\right) u d x d t\right| \\
& \quad \leq c \iint_{Q_{T}}\left[a(x)\left|v_{\varepsilon}\right|\left|\nabla v_{\varepsilon}\right|^{p(x)}+1\right] \\
& \quad \leq c .
\end{aligned}
$$

(2.9)-(2.10) yield

$$
\begin{aligned}
\left|\left\langle v_{\varepsilon t}, u\right\rangle\right| \leq & c\left[\iint_{Q_{T}}(a(x)+\varepsilon)\left(\left|v_{\varepsilon}\right|^{\alpha(x)}+\varepsilon\right)\left|\nabla v_{\varepsilon}\right|^{p(x)} d x d t\right] \\
& +c \iint_{Q_{T}}|\nabla u|^{p(x)} d x d t+c \\
\leq & c
\end{aligned}
$$

and

$$
\left\|v_{\varepsilon t}\right\|_{\mathbf{W}^{\prime}\left(Q_{T}\right)} \leq c
$$

Let $\lambda>0$ be a small enough constant, set $D_{\lambda}=\{x \in \Omega: \operatorname{dist}(x, \partial \Omega)>\lambda\}$, and let $\varphi \in C_{0}^{\infty}(\Omega)$, $0 \leq \varphi \leq 1$ satisfy

$$
\left.\varphi\right|_{D_{2 \lambda}}=1,\left.\quad \varphi\right|_{\Omega \backslash D_{\lambda}}=0
$$

Then

$$
\left|\left\langle\left(\varphi v_{\varepsilon}\right)_{t}, u\right\rangle\right|=\left|\left\langle\varphi v_{\varepsilon t}, u\right\rangle\right| \leq\left|\left\langle v_{\varepsilon t}, u\right\rangle\right|
$$

and

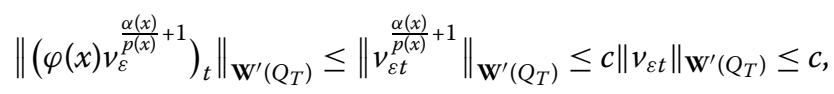

as well as

$$
\int_{\Omega}\left|\nabla\left(\varphi v_{\varepsilon}^{\frac{\alpha(x)}{p(x)}+1}\right)\right|^{p(x)} d x d t \leq c(\lambda)\left(1+\int_{D_{\lambda}}\left|\nabla v_{\varepsilon}^{\frac{\alpha(x)}{p(x)}+1}\right|^{p(x)} d x d t\right) \leq c(\lambda)
$$

or equivalently,

$$
\left\|\nabla\left(\varphi v_{\varepsilon}^{\frac{\alpha(x)}{p(x)}+1}\right)\right\|_{L^{s}\left(0, T ; L^{p(x)}(\Omega)\right)} \leq c
$$

for any $s \in(1, \infty)$. By $(2.13)-(2.14), \varphi v_{\varepsilon}^{\frac{\alpha(x)}{p(x)}+1}$ is relatively compact in $L^{s}\left(Q_{T}\right)$. Then $\varphi v_{\varepsilon}^{\frac{\alpha(x)}{p(x)}+1} \rightarrow \varphi v_{1}$ a.e. in $Q_{T}$. Due to the arbitrariness of $\lambda$, we know $v_{\varepsilon}^{\frac{\alpha(x)}{p(x)}+1} \rightarrow v_{1}$ a.e. in $Q_{T}$. 
By (2.4), $v \in L^{\infty}\left(Q_{T}\right)$, and

$$
v_{\varepsilon} \rightarrow * v, \quad \text { weakly star in } L^{\infty}\left(Q_{T}\right),
$$

it must be

$$
v_{1}=v^{\frac{\alpha(x)}{p(x)}+1} .
$$

Thus, $v_{\varepsilon} \rightarrow v$ a.e. in $Q_{T}$.

Moreover, since $a(x)$ is positive in $\Omega$, (2.8) yields

$$
\nabla v_{\varepsilon}^{\frac{\alpha(x)}{p(x)}+1} \rightarrow \nabla v^{\frac{\alpha(x)}{p(x)}+1} \quad \text { in } L^{1}\left(0, T ; L_{\text {loc }}^{p(x)}(\Omega)\right)
$$

Now, we want to show the local integral of $\nabla v$. For any $\phi(x) \in C_{0}^{1}(\Omega)$, if we choose $\left(v_{\varepsilon}^{\frac{\alpha(x)}{p(x)}+1}-v^{\frac{\alpha(x)}{p(x)}+1}\right) \phi$ as the test function, then

$$
\begin{aligned}
& \int_{0}^{T} \int_{\Omega} \frac{\partial v_{\varepsilon}}{\partial t}\left(v_{\varepsilon}^{\frac{\alpha(x)}{p(x)}+1}-v^{\frac{\alpha(x)}{p(x)}+1}\right) \phi d x d t \\
& \quad+\int_{0}^{T} \int_{\Omega} \phi(x)(a(x)+\varepsilon)\left(\left|v_{\varepsilon}\right|^{\alpha(x)}+\varepsilon\right)\left|\nabla v_{\varepsilon}\right|^{p(x)-2} \nabla v_{\varepsilon} \nabla\left(v_{\varepsilon}^{\frac{\alpha(x)}{p(x)}+1}-v^{\frac{\alpha(x)}{p(x)}+1}\right) d x d t \\
& \quad+\int_{0}^{T} \int_{\Omega}(a(x)+\varepsilon)\left(\left|v_{\varepsilon}\right|^{\alpha(x)}+\varepsilon\right)\left|\nabla v_{\varepsilon}\right|^{p(x)-2} \nabla v_{\varepsilon}\left(v_{\varepsilon}^{\frac{\alpha(x)}{p(x)}+1}-v^{\frac{\alpha(x)}{p(x)}+1}\right) \nabla \phi d x d t \\
& =\int_{0}^{T} \int_{\Omega} f\left(x, t, v_{\varepsilon}, \mid \nabla v_{\varepsilon}\right)\left(v_{\varepsilon}^{\frac{\alpha(x)}{p(x)}+1}-v^{\frac{\alpha(x)}{p(x)}+1}\right) \phi d x d t .
\end{aligned}
$$

We have the following facts:

$$
\begin{aligned}
& \lim _{\varepsilon \rightarrow 0} \int_{0}^{T} \int_{\Omega} \frac{\partial v_{\varepsilon}}{\partial t}\left(v_{\varepsilon}^{\frac{\alpha(x)}{p(x)}+1}-v^{\frac{\alpha(x)}{p(x)}+1}\right) \phi d x d t=\left\langle\frac{\partial v_{\varepsilon}}{\partial t},\left(v_{\varepsilon}^{\frac{\alpha(x)}{p(x)}+1}-v^{\frac{\alpha(x)}{p(x)}+1}\right) \phi\right\rangle=0, \\
& \lim _{\varepsilon \rightarrow 0} \int_{0}^{T} \int_{\Omega} f\left(x, t, v_{\varepsilon}, \mid \nabla v_{\varepsilon}\right)\left(v_{\varepsilon}^{\frac{\alpha(x)}{p}+1}-v^{\frac{\alpha(x)}{p(x)}+1}\right) \phi d x d t=0
\end{aligned}
$$

and

$$
\begin{aligned}
& \left.\left|\int_{0}^{T} \int_{\Omega}(a(x)+\varepsilon)\left(\left|v_{\varepsilon}\right|^{\alpha(x)}+\varepsilon\right)\right| \nabla v_{\varepsilon}\right|^{p(x)-2} \nabla v_{\varepsilon}\left(v_{\varepsilon}^{\frac{\alpha(x)}{p(x)}+1}-v^{\frac{\alpha(x)}{p(x)}+1}\right) \nabla \phi d x d t \mid \\
& \quad \leq\left\|(a(x)+\varepsilon)\left(\left|v_{\varepsilon}\right|^{\alpha(x)}+\varepsilon\right)\left|\nabla v_{\varepsilon}\right|^{p(x)-2} \nabla v_{\varepsilon}\right\|_{L^{1}\left(0, T ; L^{\frac{p(x)}{p(x)-1}}(\Omega)\right)} \\
& \quad \cdot c\left\|\nabla \phi\left(v-v_{\varepsilon}\right)\right\|_{L^{\infty}\left(0, T ; L^{p(x)}(\Omega)\right)}
\end{aligned}
$$

which goes to zero as $\varepsilon \rightarrow 0$. By (2.17)-(2.18), we can deduce that

$$
\begin{aligned}
& \lim _{\varepsilon \rightarrow 0} \int_{0}^{T} \int_{\Omega} \phi(x)(a(x)+\varepsilon)\left(\left|v_{\varepsilon}\right|^{\alpha(x)}+\varepsilon\right)\left|\nabla v_{\varepsilon}\right|^{p(x)-2} \nabla v_{\varepsilon} \nabla\left(v_{\varepsilon}^{\frac{\alpha(x)}{p(x)}+1}-v^{\frac{\alpha(x)}{p(x)}+1}\right) d x d t \\
& \quad=0,
\end{aligned}
$$


which implies that

$$
\begin{aligned}
& \left.\lim _{\varepsilon \rightarrow 0} \int_{0}^{T} \int_{\Omega} \phi(x)(a(x)+\varepsilon)\left(\left|v_{\varepsilon}\right|^{\alpha(x)}+\varepsilon\right)\right|^{\alpha(x)}\left|\nabla v_{\varepsilon}\right|^{p(x)-2} \nabla v_{\varepsilon} \nabla v^{\frac{\alpha(x)}{p(x)}+1} d x d t \\
& \quad=\lim _{\varepsilon \rightarrow 0} \int_{0}^{T} \int_{\Omega} \phi(x)(a(x)+\varepsilon)\left(\left|v_{\varepsilon}\right|^{\alpha(x)}+\varepsilon\right)\left|\nabla v_{\varepsilon}\right|^{p(x)-2} \nabla v_{\varepsilon} \nabla v_{\varepsilon}^{\frac{\alpha(x)}{p(x)}+1} d x d t \\
& \quad \leq \lim _{\varepsilon \rightarrow 0} \int_{0}^{T} \int_{\Omega} \phi(x)(a(x)+\varepsilon)\left(\left|v_{\varepsilon}\right|^{\alpha(x)}+\varepsilon\right)\left|\nabla v_{\varepsilon}\right|^{p(x)} d x d t \\
& \quad \leq c
\end{aligned}
$$

and we have

$$
\begin{aligned}
& \int_{0}^{T} \int_{\Omega} \phi(x) a(x)\left|v_{\varepsilon}\right|^{\alpha(x)}\left|\nabla v_{\varepsilon}\right|^{p(x)} \nabla v_{\varepsilon} \nabla v^{\frac{\alpha(x)}{p(x)}+1} d x d t \\
& \quad=\int_{0}^{T} \int_{\Omega}\left(\frac{\alpha(x)}{p(x)}+1\right) \phi(x) a(x)\left|v_{\varepsilon}\right|^{\alpha(x)\left(1+\frac{1}{p(x)}\right)}\left|\nabla v_{\varepsilon}\right|^{p(x)} \nabla v_{\varepsilon} \nabla v d x d t \\
& \quad \leq\left. c \int_{0}^{T} \int_{\Omega} \phi(x)(a(x)+\varepsilon)\left(\left|v_{\varepsilon}\right|^{\alpha(x)}+\varepsilon\right)\right|^{\alpha(x)}\left|\nabla v_{\varepsilon}\right|^{p(x)-2} \nabla v_{\varepsilon} \nabla v^{\frac{\alpha(x)}{p(x)}+1} d x d t \\
& \quad \leq c
\end{aligned}
$$

Since $\left|v_{\varepsilon}\right|^{\alpha(x)}\left|\nabla v_{\varepsilon}\right|^{p(x)-2} \nabla v_{\varepsilon} \in L^{1}\left(0, T ; L_{\text {loc }}^{\frac{p(x)}{p(x)}}(\Omega)\right)$, we can deduce the local integral of $\nabla v$, i.e.,

$$
\nabla v \in L^{\infty}\left(0, T ; L_{\mathrm{loc}}^{p(x)}(\Omega)\right)
$$

For any large enough $n, m, v_{n}=\left.v_{\varepsilon}\right|_{\varepsilon=\frac{1}{n}}$ and $v_{m}=\left.v_{\varepsilon}\right|_{\varepsilon=\frac{1}{m}}$ are two viscous solutions. Then

$$
\begin{aligned}
& \frac{\partial\left(v_{n}-v_{m}\right)}{\partial t} \\
& =\operatorname{div}\left[\left(a(x)+\frac{1}{n}\right)\left(\left|v_{n}\right|^{\alpha(x)}+\frac{1}{n}\right)\left|\nabla v_{n}\right|^{p(x)-2} \nabla v_{n}\right. \\
& \left.\quad-\left(a(x)+\frac{1}{m}\right)\left(\left|v_{m}\right|^{\alpha(x)}+\frac{1}{m}\right)\left|\nabla v_{m}\right|^{p(x)-2} \nabla v_{m}\right] \\
& \quad+\left[f\left(x, t, v_{n},\left|\nabla v_{n}\right|\right)-f\left(x, t, v_{m},\left|\nabla u_{m}\right|\right)\right] .
\end{aligned}
$$

Egoroff's theorem yields, for fixed $\delta>0$, a closed set $E_{\delta} \subset Q_{T}$ such that the measure $\mu\left(Q_{T}-E_{\delta}\right) \leq \delta$ and $v_{n} \rightrightarrows v$ uniformly on $E_{\delta}$. By drawing the methods of [26-28], we can extrapolate that

$$
\iint_{E_{\delta}} a(x)|v|^{\alpha(x)}\left|\nabla v_{n}-\nabla v_{m}\right|^{p} d x d t \rightarrow 0
$$

from which we can deduce $\nabla v_{n} \rightarrow \nabla v$ a.e. in $Q_{T}$. Thus, we have

$$
v_{\varepsilon} \rightarrow * v \quad \text { in } L^{\infty}\left(Q_{T}\right),
$$




$$
(a(x)+\varepsilon)\left(\left|v_{\varepsilon}\right|^{\alpha}+\varepsilon\right)\left|\nabla v_{\varepsilon}\right|^{p(x)-2} \nabla v_{\varepsilon} \rightarrow a(x)|v|^{\alpha(x)}|\nabla v|^{p(x)-2} \nabla u \quad \text { in } L^{p^{-}}\left(0, T ; L^{\frac{p(x)}{p(x)-1}}(\Omega)\right) .
$$

In the end, the initial value is true in the sense of (1.14) can be shown as that of [1]. Thus, $v$ is a weak solution of equation (1.1) in the sense of Definition 1.1.

\section{The global stability}

For small $\eta>0$, we define $g_{\eta}(x)$ to be an odd function, when $s \geq 0, g_{\eta}(x)$ has the form

$$
\begin{aligned}
& g_{\eta}(s)= \begin{cases}1, & s \geq \eta, \\
\frac{s^{2}}{\eta^{2}} \mathrm{e}^{1-\frac{s^{2}}{\eta^{2}},}, & 0 \leq s \leq \eta,\end{cases} \\
& G_{\eta}(s)=\int_{0}^{s} g_{\eta}(s) d s, \\
& \lim _{\eta \rightarrow 0} g_{\eta}(s)=\operatorname{sgn} s, \quad \lim _{\eta \rightarrow 0} s g_{\eta}^{\prime}(s)=0, \quad \lim _{\eta \rightarrow 0} G_{\eta}(s)=|s| .
\end{aligned}
$$

Proceeding as in [28], we can prove the following lemma, we omit the details here.

Lemma 3.1 Let $u \in \mathbf{W}\left(Q_{T}\right), u_{t} \in \mathbf{W}^{\prime}\left(Q_{T}\right)$. Then $\forall$ a.e. $t_{1}, t_{2} \in(0, T)$,

$$
\int_{t_{1}}^{t_{2}} \int_{\Omega} g_{\eta}(u) u_{t} d x d t=\left[\int_{\Omega}\left[G_{\eta}(u)\left(x, t_{2}\right)-G_{\eta}(u)\left(x, t_{1}\right)\right] d x\right] .
$$

The following lemma is the basic characteristics of the variable exponent Sobolev spaces $[6,12,32]$.

\section{Lemma 3.2}

(i) The spaces $\left(L^{p(x)}(\Omega),\|\cdot\|_{L^{p(x)}(\Omega)}\right),\left(W^{1, p(x)}(\Omega),\|\cdot\|_{W^{1, p(x)}(\Omega)}\right)$, and $W_{0}^{1, p(x)}(\Omega)$ are reflexive Banach spaces.

(ii) The $p(x)$-Hölder inequality. Let $p(x)$ and $q(x)$ be real functions with $\frac{1}{p(x)}+\frac{1}{q(x)}=1$. Then, for any $u \in L^{p(x)}(\Omega)$ and $v \in L^{q(x)}(\Omega)$, we have

$$
\left|\int_{\Omega} u v d x\right| \leq 2\|u\|_{L^{p(x)}(\Omega)}\|v\|_{L^{q(x)}(\Omega)}
$$

(iii) $\|u\|_{L^{p(x)}(\Omega)}$ and $\int_{\Omega}|u|^{p(x)} d x$ satisfy

$$
\begin{aligned}
& \text { If }\|u\|_{L^{p(x)}(\Omega)}=1, \quad \text { then } \int_{\Omega}|u|^{p(x)} d x=1 . \\
& \text { If }\|u\|_{L^{p(x)}(\Omega)}>1, \quad \text { then }\|u\|_{L^{p(x)}(\Omega)}^{p^{-}} \leq \int_{\Omega}|u|^{p(x)} d x \leq\|u\|_{L^{p(x)}(\Omega)}^{p^{+}} \\
& \text {If }\|u\|_{L^{p(x)}(\Omega)}<1, \quad \text { then }\|u\|_{L^{p(x)}(\Omega)}^{p^{+}} \leq \int_{\Omega}|u|^{p(x)} d x \leq\|u\|_{L^{p(x)}(\Omega)}^{p^{-}}
\end{aligned}
$$

Theorem 3.3 Let $u(x, t)$ and $v(x, t)$ be two solutions of equation (1.1) with the initial values $u_{0}(x)$ and $v_{0}(x)$ respectively and with the same homogeneous boundary value condition (1.18). If $\alpha(x) \in C_{0}^{1}(\Omega)$, the nonlinear damping term satisfies 
(A) $p^{-} \geq 2$,

$$
\begin{aligned}
& |f(x, t, u,|\nabla u|)-f(x, t, u,|\nabla v|)| \\
& \quad \leq c f_{1}(x, t)|u-v|\left[|v|^{\frac{2 \alpha(x)}{p(x)}}|\nabla v|^{2}+|u|^{\frac{2 \alpha(x)}{p(x)}}|\nabla u|^{2}\right],
\end{aligned}
$$

(B) $p^{-}>1$,

$$
\begin{aligned}
& |f(x, t, u,|\nabla u|)-f(x, t, u,|\nabla v|)| \\
& \quad \leq c f_{1}(x, t)|u-v|\left[|v|^{\frac{\alpha(x)}{p(x)}}|\nabla v|+|u|^{\frac{2 \alpha(x)}{p(x)}}|\nabla u|\right],
\end{aligned}
$$

and one of the following conditions is true:

(i)

$$
a(x)^{-1} f_{1}(x, t) \leq c
$$

(ii) there is a constant $r \geq 2+\frac{2}{p(x)-2}$ such that

$$
\iint_{Q_{T}} a(x)^{1-r} f_{1}(x, t)^{r} d x d t \leq c
$$

then

$$
\int_{\Omega}|u(x, t)-v(x, t)| d x \leq c \int_{\Omega}\left|u_{0}(x)-v_{0}(x)\right| d x, \quad \text { a.e. } t \in[0, T) .
$$

Proof We only give the proof of case (A). Case (B) can be proved in a similar way, we omit the details.

Since $u(x, t)$ and $v(, t)$ satisfy the same homogeneous boundary value condition (1.18), we can choose $g_{\eta}(u-v)$ as the test function. Then

$$
\begin{aligned}
& \int_{0}^{t} \int_{\Omega} \frac{\partial(u-v)}{\partial t} g_{\eta}(u-v) d x d t \\
& \quad+\int_{0}^{t} \int_{\Omega} a(x)|u|^{\alpha(x)}\left(|\nabla u|^{p(x)-2} \nabla u-|\nabla v|^{p(x)-2} \nabla v\right) \nabla(u-v) g_{\eta}^{\prime}(u-v) d x d t \\
& \quad+\int_{0}^{t} \int_{\Omega} a(x)\left(|u|^{\alpha(x)}-|v|^{\alpha(x)}\right)|\nabla v|^{p(x)-2} \nabla v \nabla(u-v) g_{\eta}^{\prime}(u-v) d x d t \\
& =\int_{0}^{t} \int_{\Omega}[f(x, t, u,|\nabla u|)-f(x, t, v,|\nabla v|)] g_{\eta}(u-v) d x d t .
\end{aligned}
$$

There are two facts much in evidence in (3.5). One is that, by Lemma 3.1, we have

$$
\begin{aligned}
& \lim _{\eta \rightarrow 0} \int_{0}^{t} \int_{\Omega} g_{\eta}(u-v) \frac{\partial(u-v)}{\partial t} d x d t \\
& \quad=\int_{\Omega}|u(x, t)-v(x, t)| d x-\int_{\Omega}\left|u_{0}(x)-v_{0}(x)\right| d x .
\end{aligned}
$$


Another one is that, by the monotonicity of the operator $|\nabla u|^{r-2} \nabla u$, we have

$$
\int_{\Omega} a(x)|u|^{\alpha(x)}\left(|\nabla u|^{p(x)-2} \nabla u-|\nabla v|^{p(x)-2} \nabla v\right) \nabla(u-v) g_{\eta}^{\prime}(u-v) d x \geq 0 .
$$

Let us discuss the other terms in (3.5). In the first place, $\alpha(x) \in C_{0}^{1}(\Omega)$, we set $\Omega_{\alpha}=\{x \in$ $\Omega: \alpha(x)>0\}$ and define

$$
\begin{aligned}
& D_{t}=\{x \in \Omega: u(x, t) \neq v(x, t)\}, \\
& D_{1 t}=\Omega_{\alpha} \cap D_{t}, \quad D_{2 t}=\left(\Omega \backslash \Omega_{\alpha}\right) \cap D_{t} .
\end{aligned}
$$

Since (2.23) yields $|\nabla u|^{p(x)},|\nabla v|^{p(x)} \in L_{\text {loc }}^{1}\left(Q_{T}\right)$, using the fact $\lim _{\eta \rightarrow 0} g_{\eta}^{\prime}(s) s=0$ and the Lebesgue dominated convergence theorem, we have

$$
\begin{aligned}
& \left.\lim _{\eta \rightarrow 0} \int_{0}^{t} \int_{\Omega} a(x)|| u\right|^{\alpha(x)}-\left.|v|^{\alpha(x)}|| \nabla v\right|^{p(x)} g_{\eta}^{\prime}(u-v) d x d t \\
& \quad=\left.\lim _{\eta \rightarrow 0} \int_{0}^{t} \int_{D_{1 t}} a(x)|| u\right|^{\alpha(x)}-\left.|v|^{\alpha(x)}|| \nabla v\right|^{p(x)} g_{\eta}^{\prime}(u-v) d x d t \\
& \quad=\int_{0}^{t} \int_{D_{1 t}} \lim _{\eta \rightarrow 0} a(x) \alpha(x)|\xi|^{\alpha(x)-1}|u-v||\nabla v|^{p(x)} g_{\eta}^{\prime}(u-v) d x d t \\
& \quad=0
\end{aligned}
$$

and similarly

$$
\left.\lim _{\eta \rightarrow 0} \int_{0}^{t} \int_{\Omega} a(x)|| u\right|^{\alpha(x)}-\left.|v|^{\alpha(x)}|| \nabla u\right|^{p} g_{\eta}^{\prime}(u-v) d x=0 .
$$

According to (3.8)-(3.9), we can obtain

$$
\begin{aligned}
& \left.\lim _{\eta \rightarrow 0}\left|\int_{0}^{t} \int_{\Omega} a(x)\left(|u|^{\alpha(x)}-|v|^{\alpha(x)}\right)\right| \nabla v\right|^{p(x)-2} \nabla v \nabla(u-v) g_{\eta}^{\prime}(u-v) d x d t \mid \\
& \leq\left.\lim _{\eta \rightarrow 0} \int_{0}^{t} \int_{D_{1 t}} a(x)|| u\right|^{\alpha(x)}-|v|^{\alpha(x)}|||\nabla v|^{p(x)-1}(|\nabla u|+|\nabla v|) g_{\eta}^{\prime}(u-v) d x d t \\
& \leq\left. c \lim _{\eta \rightarrow 0} \int_{0}^{t} \int_{D_{1 t}} a(x)|| u\right|^{\alpha(x)}-\left.|v|^{\alpha(x)}|| \nabla v\right|^{p(x)-1}|\nabla u| g_{\eta}^{\prime}(u-v) d x d t \\
& \quad+\left.\lim _{\eta \rightarrow 0} \int_{0}^{t} \int_{D_{1 t}} a(x)|| u\right|^{\alpha(x)}-|v|^{\alpha(x)}|||\nabla v|^{p(x)} g_{\eta}^{\prime}(u-v) d x d t \\
& \leq c \lim _{\eta \rightarrow 0}\left(\left.\int_{0}^{t} \int_{D_{1 t}} a(x)|| u\right|^{\alpha(x)}-|v|^{\alpha(x)}|||\nabla v|^{p(x)} g_{\eta}^{\prime}(u-v) d x d t\right)^{\frac{1}{q^{+}}} \\
& \quad \cdot\left(\left.\int_{0}^{t} \int_{D_{1 t}} a(x)|| u\right|^{\alpha(x)}-\left.|v|^{\alpha(x)}|| \nabla u\right|^{p(x)} g_{\eta}^{\prime}(u-v) d x d t\right)^{\frac{1}{p^{+}}} \\
& \quad+\left.\left.\lim _{\eta \rightarrow 0} \int_{0}^{t} \int_{D_{1 t}} a(x)|| \nabla v\right|^{p(x)}|| u\right|^{\alpha(x)}-|v|^{\alpha(x)}|| g_{\eta}^{\prime}(u-v) d x d t \\
& =0,
\end{aligned}
$$

where $p^{+}$and $q^{+}$follow from (iii) of Lemma 3.2. 
In the second place, since the nonlinear damping term satisfies (3.1), using the Hölder inequality, we have:

(i) By (3.3),

$$
\begin{aligned}
\mid \lim _{\eta \rightarrow 0} & \int_{0}^{t} \int_{\Omega}[f(x, t, u,|\nabla u|)-f(x, t, v,|\nabla v|)] g_{\eta}(u-v) d x d t \mid \\
\leq & c \int_{0}^{t} \int_{\Omega} a(x)\left[|v|^{\frac{2 \alpha(x)}{p(x)}}|\nabla v|^{2}+|u|^{\frac{2 \alpha(x)}{p(x)}}|\nabla u|^{2}\right]\left|a(x)^{-1} f_{1}(x, t)\right||u-v| d x d t \\
\leq & c\left(\int_{0}^{t} \int_{\Omega} a(x)\left[|u|^{\alpha(x)}|\nabla u|^{p(x)}+|v|^{\alpha(x)}|\nabla v|^{p(x)}\right] d x d t\right)^{\frac{2}{p_{21}}} \\
& \cdot\left(\int_{0}^{t} \int_{\Omega} a(x)|u-v|^{\frac{p(x)}{p(x)-2}} d x d t\right)^{\frac{1}{p_{22}}} \\
\leq & c\left(\int_{0}^{t} \int_{\Omega}|u-v|^{\frac{p(x)}{p(x)-2}} d x d t\right)^{\frac{1}{p_{22}}} \\
\leq & c\left(\int_{0}^{t} \int_{\Omega}|u(x, t)-v(x, t)| d x d t\right)^{\frac{1}{p_{22}}}
\end{aligned}
$$

where $p_{21}=\max _{x \in \bar{\Omega}} \frac{p(x)}{2}$ or $\min _{x \in \bar{\Omega}} \frac{p(x)}{2}$ according to (iii) of Lemma 3.2, $p_{22}$ has a similar sense.

(ii) Since $r \geq 2+\frac{2}{p(x)-2}$, there is $\frac{p(x)}{p(x)-2} \frac{(r-1) p(x)-2 r}{p(x)} \geq 1$. By (3.4), there are two constants $l_{1}>1, l_{2}>1$ such that

$$
\begin{aligned}
\lim _{\eta \rightarrow 0} & \int_{0}^{t} \int_{\Omega}[f(x, t, u,|\nabla u|)-f(x, t, v,|\nabla v|)] g_{\eta}(u-v) d x d t \mid \\
\leq & c \int_{0}^{t} \int_{\Omega}\left[|v|^{\frac{2 \alpha(x)}{p(x)}}|\nabla v|^{2}+|u|^{\frac{2 \alpha(x)}{p(x)}}|\nabla u|^{2}\right]\left|f_{1}(x, t)\right||u-v| d x d t \\
\leq & \left(\int_{0}^{t} \int_{\Omega} a(x)\left[|u|^{\alpha(x)}|\nabla u|^{p(x)}+|v|^{\alpha(x)}|\nabla v|^{p(x)}\right] d x d t\right)^{\frac{2}{p_{21}}} \\
& \cdot\left(\int_{0}^{t} \int_{\Omega} a(x)\left|a(x)^{-1} f_{1}(x, t)(u-v)\right|^{\frac{p(x)}{p(x)-2}} d x d t\right)^{\frac{1}{p_{22}}} \\
\leq & \left(\int_{0}^{t} \int_{\Omega} a(x)\left|a(x)^{-1} f_{1}(x, t)\right|^{\frac{p(x)}{p(x)-2}} \frac{(p(x)-2) r}{p(x)} d x d t\right)^{\frac{1}{l_{1}}} \\
& \cdot\left(\int_{0}^{t} \int_{\Omega}|u-v|^{\frac{p(x)}{p(x)-2} \frac{(r-1) p(x)-2 r}{p(x)}} d x d t\right)^{\frac{1}{l_{2}}} \\
\leq & c\left(\int_{0}^{t} \int_{\Omega}|u(x, t)-v(x, t)| d x d t\right)^{\frac{1}{l_{2}}} .
\end{aligned}
$$

Now, let $\eta \rightarrow 0$ in (3.5). According to (3.11)-(3.12), there is a constant $l_{3}>1$ such that

$$
\int_{\Omega}|u(x, t)-v(x, t)| d x \leq \int_{\Omega}\left|u_{0}(x)-v_{0}(x)\right| d x+c\left(\int_{0}^{t} \int_{\Omega}|u(x, t)-v(x, t)| d x d t\right)^{\frac{1}{l_{3}}} .
$$

By a generalized Gronwall inequality [26], we have the conclusion. 
Proof of Theorem 1.3 If the nonlinear damping term satisfies (1.19)

$$
\begin{aligned}
& |f(x, t, u,|\nabla u|)-f(x, t, v,|\nabla v|)| \\
& \quad \leq c\left[(a(x)|v|)^{\frac{2 \alpha(x)}{p(x)}}|\nabla v|^{2}+(a(x)|u|)^{\frac{2 \alpha(x)}{p(x)}}|\nabla u|^{2}\right]|u-v|,
\end{aligned}
$$

we easily show that there is a constant $l>1$ such that

$$
\begin{aligned}
& \left|\lim _{\eta \rightarrow 0} \int_{0}^{t} \int_{\Omega}[f(x, t, u,|\nabla u|)-f(x, t, v,|\nabla v|)] g_{\eta}(u-v) d x d t\right| \\
& \quad \leq c\left(\int_{0}^{t} \int_{\Omega}|u(x, t)-v(x, t)| d x d t\right)^{\frac{1}{T}} .
\end{aligned}
$$

Proceeding as in the proof of Theorem 3.3, we have the conclusion. If the nonlinear damping term satisfies (1.20), we can prove the conclusion in a similar way, and we do not repeat the details here.

Theorem 3.4 Let $p^{-} \geq 2, u(x, t)$ and $v(x, t)$ be two solutions of equation (1.1) with the initial values $u_{0}(x)$ and $v_{0}(x)$ respectively and with the same homogeneous boundary value condition (1.18). If $\alpha(x) \equiv 0$, the nonlinear damping term satisfies

$$
|f(x, t, u,|\nabla u|)-f(x, t, u,|\nabla v|)| \leq c f_{1}(x, t)\left(|\nabla v|^{2}+|\nabla u|^{2}\right)
$$

and condition (3.3) or (3.4) is true, then

$$
\int_{\Omega}|u(x, t)-v(x, t)|^{2} d x \leq c \int_{\Omega}\left|u_{0}(x)-v_{0}(x)\right|^{2} d x, \quad \text { a.e. } t \in[0, T) .
$$

Proof Since $u(x, t)$ and $v(, t)$ satisfy the same homogeneous boundary value condition (1.18), we can choose $(u-v)$ as the test function. Then

$$
\begin{aligned}
& \int_{0}^{t} \int_{\Omega} \frac{\partial(u-v)}{\partial t}(u-v) d x d t \\
& \quad+\int_{0}^{t} \int_{\Omega} a(x)|u|^{\alpha(x)}\left(|\nabla u|^{p(x)-2} \nabla u-|\nabla v|^{p(x)-2} \nabla v\right) \nabla(u-v) d x d t \\
& \quad=\int_{0}^{t} \int_{\Omega}[f(x, t, u,|\nabla u|)-f(x, t, v,|\nabla v|)](u-v) d x d t
\end{aligned}
$$

By Lemma 3.1, we have

$$
\begin{aligned}
& \int_{0}^{t} \int_{\Omega}(u-v) \frac{\partial(u-v)}{\partial t} d x d t \\
& \quad=\frac{1}{2} \int_{\Omega}|u(x, t)-v(x, t)| d x-\frac{1}{2} \int_{\Omega}\left|u_{0}(x)-v_{0}(x)\right| d x
\end{aligned}
$$

and

$$
\int_{\Omega} a(x)\left(|\nabla u|^{p(x)-2} \nabla u-|\nabla v|^{p(x)-2} \nabla v\right) \nabla(u-v) d x \geq 0 .
$$


At the same time, since the nonlinear damping term satisfies (3.14), using the Hölder inequality, we have the following:

(i) By (3.3), there is a constant $l>1$ such that

$$
\begin{aligned}
& \left|\int_{0}^{t} \int_{\Omega}[f(x, t, u,|\nabla u|)-f(x, t, v,|\nabla v|)](u-v) d x d t\right| \\
& \leq c \int_{0}^{t} \int_{\Omega} a(x)\left(|\nabla v|^{2}+|\nabla u|^{2}\right)\left|a(x)^{-1} f_{1}(x, t)\right||u-v| d x d t \\
& \leq c\left(\int_{0}^{t} \int_{\Omega} a(x)\left(|\nabla u|^{p(x)}+|\nabla v|^{p(x)}\right) d x d t\right)^{\frac{2}{p_{21}}} \\
& \quad \cdot\left(\int_{0}^{t} \int_{\Omega} a(x)|u-v|^{\frac{p(x)}{p(x)-2}} d x d t\right)^{\frac{1}{p_{22}}} \\
& \leq c\left(\int_{0}^{t} \int_{\Omega}|u-v|^{\frac{p(x)}{p(x)-2}} d x d t\right)^{\frac{1}{p_{22}}} \\
& \leq c\left(\int_{0}^{t} \int_{\Omega}|u(x, t)-v(x, t)|^{2} d x d t\right)^{\frac{1}{l}},
\end{aligned}
$$

where $p_{21}=\max _{x \in \bar{\Omega}} \frac{p(x)}{2}$ or $\min _{x \in \bar{\Omega}} \frac{p(x)}{2}$ according to (iii) of Lemma 3.2, $p_{22}$ has a similar sense.

(ii) Since $r \geq 2+\frac{2}{p(x)-2}$, there is $\frac{p(x)}{p(x)-2} \frac{(r-1) p(x)-2 r}{p(x)} \geq 1$. By (3.14), there are constants $l_{1}>1$, $l_{2}>1$, and $l_{3}>1$ such that

$$
\begin{aligned}
\left|\int_{0}^{t} \int_{\Omega}[f(x, t, u,|\nabla u|)-f(x, t, v,|\nabla v|)](u-v) d x d t\right| \\
\leq c \int_{0}^{t} \int_{\Omega}\left(|\nabla v|^{2}+|\nabla u|^{2}\right)\left|f_{1}(x, t)\right||u-v| d x d t \\
\leq\left(\int_{0}^{t} \int_{\Omega} a(x)\left(|\nabla u|^{p(x)}+|\nabla v|^{p(x)}\right) d x d t\right)^{\frac{2}{p_{21}}} \\
\cdot\left(\int_{0}^{t} \int_{\Omega} a(x)\left|a(x)^{-1} f_{1}(x, t)(u-v)\right|^{\frac{p(x)}{p(x)-2}} d x d t\right)^{\frac{1}{p_{22}}} \\
\leq c\left(\int_{0}^{t} \int_{\Omega} a(x)\left|a(x)^{-1} f_{1}(x, t)\right|^{\frac{p(x)}{p(x)-2}} \frac{(p(x)-2) r}{p(x)} d x d t\right)^{\frac{1}{l_{1}}} \\
\quad \cdot\left(\int_{0}^{t} \int_{\Omega}|u-v|^{\frac{p(x)}{p(x)-2}} \frac{(r-1) p(x)-2 r}{p(x)} d x d t\right)^{\frac{1}{l_{2}}} \\
\leq c\left(\int_{0}^{t} \int_{\Omega}|u(x, t)-v(x, t)|^{2} d x d t\right)^{\frac{1}{l_{3}}} .
\end{aligned}
$$

According to (3.18)-(3.19), there is a constant $l_{4}>1$ such that

$$
\int_{\Omega}|u(x, t)-v(x, t)|^{2} d x \leq \int_{\Omega}\left|u_{0}(x)-v_{0}(x)\right|^{2} d x+c\left(\int_{0}^{t} \int_{\Omega}|u(x, t)-v(x, t)|^{2} d x d t\right)^{\frac{1}{l_{4}}} .
$$

By a generalized Gronwall inequality [26], we have the conclusion. 
Proof of Theorem 1.4 Since the nonlinear damping term satisfies

$$
\begin{aligned}
& |f(x, t, u,|\nabla u|)-f(x, t, v,|\nabla v|)| \\
& \quad \leq c a(x)\left(|\nabla v|^{2}+|\nabla u|^{2}\right),
\end{aligned}
$$

proceeding as in the proof of Theorem 3.4, we have the conclusion.

\section{The global stability if $\int_{\Omega} a(x)^{1-p(x)} d x<\infty$}

Recalling that, by a weak characteristic function $\chi(x)$ of $\Omega, \chi(x) \in C(\bar{\Omega})$ and

$$
\chi(x)=0, \quad x \in \partial \Omega, \quad \chi(x)>0, \quad x \in \Omega,
$$

we can set another weak characteristic function as

$$
\chi_{\lambda}(x)= \begin{cases}1, & \chi(x) \geq \lambda \\ \frac{\chi(x)}{\lambda}, & \chi(x) \leq \lambda\end{cases}
$$

In this section, we explore the stability of weak solutions by the weak characteristic function method [29, 30].

Theorem 4.1 Suppose that $u(x, t)$ and $v(x, t)$ are two solutions of equation (1.2) with the initial values $u_{0}(x), v_{0}(x)$ respectively. If there is a weak characteristic function $\chi(x) \in C^{1}(\bar{\Omega})$ satisfying

$$
\frac{1}{\lambda}\left(\int_{\Omega \backslash \Omega_{\lambda}} a(x)|\nabla \chi|^{p(x)} d x\right)^{\frac{1}{p^{+}}}<\infty
$$

$\alpha(x) \in C_{0}^{1}(\Omega)$, the nonlinear damping term satisfies (3.1) and one of (3.3) (3.4), then

$$
\int_{\Omega}|u(x, t)-v(x, t)| d x \leq c \int_{\Omega}\left|u_{0}(x)-v_{0}(x)\right| d x, \quad \text { a.e. } t \in[0, T) .
$$

Proof Since $\alpha(x) \in C_{0}^{1}(\Omega)$, as before we set $\Omega_{\alpha}=\{x \in \Omega: \alpha(x)>0\}$ and

$$
D_{1 t}=\Omega_{\alpha} \cap\{x \in \Omega: u(x, t) \neq v(x, t)\}, \quad D_{2 t}=\left(\Omega \backslash \Omega_{\alpha}\right) \cap\{x \in \Omega: u(x, t) \neq v(x, t)\},
$$

as well as

$$
D_{t}=\{x \in \Omega: u(x, t) \neq v(x, t)\} .
$$


By choosing $g_{\eta}(u-v) \phi_{\lambda}(x)$ as a test function, since

$$
\begin{aligned}
& \iint_{Q_{t}} a(x) \chi_{\lambda}(x)\left[|u|^{\alpha(x)}|\nabla u|^{p(x)-2} \nabla u-|v|^{\alpha(x)}|\nabla v|^{p(x)-2} \nabla v\right] \nabla(u-v) g_{\eta}^{\prime}(u-v) d x d t \\
& \quad=\int_{0}^{t} \int_{D_{t}} a(x) \chi_{\lambda}(x)\left[|u|^{\alpha(x)}|\nabla u|^{p(x)-2} \nabla u-|v|^{\alpha(x)}|\nabla v|^{p(x)-2} \nabla v\right] \nabla(u-v) g_{\eta}^{\prime}(u-v) d x d t \\
& \quad=\int_{0}^{t} \int_{D_{1 t}} a(x) \chi_{\lambda}(x)\left[|u|^{\alpha(x)}|\nabla u|^{p(x)-2} \nabla u-|v|^{\alpha(x)}|\nabla v|^{p(x)-2} \nabla v\right] \nabla(u-v) g_{\eta}^{\prime}(u-v) d x d t \\
& \quad+\int_{0}^{t} \int_{D_{2 t}} a(x) \chi_{\lambda}(x)\left[|\nabla u|^{p(x)-2} \nabla u-|\nabla v|^{p(x)-2} \nabla v\right] \nabla(u-v) g_{\eta}^{\prime}(u-v) d x d t,
\end{aligned}
$$

we have

$$
\begin{aligned}
\int_{0}^{t} \int_{\Omega} & \frac{\partial(u-v)}{\partial t} \chi_{\lambda}(x) g_{\eta}(u-v) d x d t \\
& +\int_{0}^{t} \int_{D_{1 t}} a(x) \chi_{\lambda}(x)|u|^{\alpha(x)}\left(|\nabla u|^{p(x)-2} \nabla u-|\nabla v|^{p(x)-2} \nabla v\right) \nabla(u-v) g_{\eta}^{\prime}(u-v) d x d t \\
& +\int_{0}^{t} \int_{D_{1 t}} a(x) \chi_{\lambda}(x)\left(|u|^{\alpha(x)}-|v|^{\alpha(x)}\right)|\nabla v|^{p(x)-2} \nabla v \nabla(u-v) g_{\eta}^{\prime}(u-v) d x d t \\
& +\int_{0}^{t} \int_{D_{2 t}} a(x) \chi_{\lambda}(x)\left[|\nabla u|^{p(x)-2} \nabla u-|\nabla v|^{p(x)-2} \nabla v\right] \nabla(u-v) g_{\eta}^{\prime}(u-v) d x d t \\
& +\int_{0}^{t} \int_{D_{1 t}} a(x)|u|^{\alpha(x)}\left(|\nabla u|^{p(x)-2} \nabla u-|\nabla v|^{p(x)-2} \nabla v\right) \nabla \chi_{\lambda} g_{\eta}(u-v) d x d t \\
& +\int_{0}^{t} \int_{D_{1 t}} a(x)\left(|u|^{\alpha(x)}-|v|^{\alpha(x)}\right)|\nabla v|^{p(x)-2} \nabla v \nabla \chi_{\lambda} g_{\eta}(u-v) d x d t \\
& +\int_{0}^{t} \int_{D_{2 t}} a(x)\left(|\nabla u|^{p(x)-2} \nabla u-|\nabla v|^{p(x)-2} \nabla v\right) \nabla \chi_{\lambda}(x) g_{\eta}(u-v) d x d t \\
= & \int_{0}^{t} \int_{\Omega}[f(x, t, u,|\nabla u|)-f(x, t, v,|\nabla v|)] \chi_{\lambda}(x) g_{\eta}((u-v)) d x d t \\
\leq & \int_{0}^{t} \int_{D_{1 t}} f_{1}(x, t)|u-v|\left[|v|^{\frac{2 \alpha(x)}{p(x)}}|\nabla v|^{2}+|u|^{\frac{2 \alpha(x)}{p(x)}}|\nabla u|^{2}\right] d x d t .
\end{aligned}
$$

As usual, we now analyze every term in (4.4). In the first place, we have

$$
\begin{aligned}
& \lim _{\lambda \rightarrow 0} \int_{0}^{t} \int_{\Omega} \chi_{\lambda} \frac{\partial G_{\eta}(u-v)}{\partial t} d x d t \\
& \quad=\int_{\Omega}|u(x, t)-v(x, t)| d x-\int_{\Omega}\left|u_{0}(x)-v_{0}(x)\right| d x
\end{aligned}
$$

and

$$
\begin{aligned}
& \int_{0}^{t} \int_{D_{1 t}} \chi_{\lambda}(x) a(x)|u|^{\alpha(x)}\left(|\nabla u|^{p(x)-2} \nabla u-|\nabla v|^{p(x)-2} \nabla v\right) \nabla(u-v) g_{\eta}^{\prime}(u-v) d x d t \geq 0, \\
& \int_{0}^{t} \int_{D_{2 t}} \chi_{\lambda}(x) a(x)\left(|\nabla u|^{p(x)-2} \nabla u-|\nabla v|^{p(x)-2} \nabla v\right) \nabla(u-v) g_{\eta}^{\prime}(u-v) d x \geq 0
\end{aligned}
$$


In the second place, since $|\nabla u|^{p(x)},|\nabla v|^{p(x)} \in L_{\text {loc }}^{1}\left(Q_{T}\right)$, using the fact $\lim _{\eta \rightarrow 0} g_{\eta}^{\prime}(s) s=0$ and the Lebesgue dominated convergence theorem, we have

$$
\begin{aligned}
& \left.\lim _{\eta \rightarrow 0} \lim _{\lambda \rightarrow 0} \int_{0}^{t} \int_{D_{1 t}} \chi_{\lambda}(x) a(x)|| u\right|^{\alpha(x)}-\left.|v|^{\alpha(x)}|| \nabla u\right|^{p(x)} g_{\eta}^{\prime}(u-v) d x=0, \\
& \left.\lim _{\eta \rightarrow 0} \lim _{\lambda \rightarrow 0} \int_{0}^{t} \int_{D_{1 t}} \chi_{\lambda}(x) a(x)|| u\right|^{\alpha(x)}-\left.|v|^{\alpha(x)}|| \nabla v\right|^{p(x)} g_{\eta}^{\prime}(u-v) d x=0,
\end{aligned}
$$

thus

$$
\begin{aligned}
&\left.\lim _{\eta \rightarrow 0} \lim _{\lambda \rightarrow 0}\left|\int_{D_{1 t}} \chi_{\lambda}(x) a(x)\left(|u|^{\alpha(x)}-|v|^{\alpha(x)}\right)\right| \nabla v\right|^{p(x)-2} \nabla v \nabla(u-v) g_{\eta}^{\prime}(u-v) d x d t \mid \\
&=\left.\lim _{\eta \rightarrow 0}\left|\int_{0}^{t} \int_{D_{1 t}} a(x)\left(|u|^{\alpha(x)}-|v|^{\alpha(x)}\right)\right| \nabla v\right|^{p(x)-2} \nabla v \nabla(u-v) g_{\eta}^{\prime}(u-v) d x d t \mid \\
& \leq\left.\lim _{\eta \rightarrow 0} \int_{0}^{t} \int_{D_{1 t}} a(x)|| u\right|^{\alpha(x)}-|v|^{\alpha(x)}|||\nabla v|^{p(x)-1}(|\nabla u|+|\nabla v|) g_{\eta}^{\prime}(u-v) d x d t \\
& \leq\left. c \lim _{\eta \rightarrow 0} \int_{0}^{t} \int_{D_{1 t}} a(x)|| u\right|^{\alpha(x)}-\left.|v|^{\alpha(x)}|| \nabla v\right|^{p(x)-1}|\nabla u| g_{\eta}^{\prime}(u-v) d x d t \\
& \quad+\left.\int_{0}^{t} \int_{D_{1 t}} a(x)|| u\right|^{\alpha(x)}-|v|^{\alpha(x)}|||\nabla v|^{p(x)} g_{\eta}^{\prime}(u-v) d x d t \\
& \leq c \lim _{\eta \rightarrow 0} \int_{0}^{t}\left(\left.\int_{D_{1 t}} a(x)|| u\right|^{\alpha(x)}-|v|^{\alpha(x)}|||\nabla v|^{p(x)} g_{\eta}^{\prime}(u-v) d x\right)^{\frac{1}{q_{1}}} \\
& \cdot\left(\left.\int_{D_{1 t}} a(x)|| u\right|^{\alpha(x)}-\left.|v|^{\alpha(x)}|| \nabla u\right|^{p(x)} g_{\eta}^{\prime}(u-v) d x\right)^{\frac{1}{p_{1}}} d t \\
&+\left.\left.\lim _{\eta \rightarrow 0} \int_{0}^{t} \int_{D_{1 t}} a(x)|| \nabla v\right|^{p(x)}|| u\right|^{\alpha(x)}-|v|^{\alpha(x)}|| g_{\eta}^{\prime}(u-v) d x d t \\
&= 0 .
\end{aligned}
$$

Here, $p_{1}$ is $p^{+}$or $p^{-}$according to (iii) of Lemma 3.2, $q_{1}$ is $q^{+}$or $q^{-}$.

In the third place, we denote $D_{\lambda}=\{x \in \Omega: \chi(x)>\lambda\}$. If we choose $\lambda$ small enough, then for $\Omega_{\alpha}=\{x \in \Omega: \alpha(x)>0\}$,

$$
\Omega_{\alpha} \subset D_{\lambda}, \quad \Omega \backslash D_{\lambda} \subset \Omega \backslash \Omega_{\alpha},
$$

there is a constant $c_{\alpha}$ such that $\phi(x)>c_{\alpha}$ provided $x \in \bar{\Omega}_{\alpha}$. According to the definition of the weak characteristic function $\chi(x)$,

$$
\nabla \chi_{\lambda}(x)=0, \quad x \in \Omega_{\alpha}
$$

If we define that

$$
\begin{aligned}
& D_{3 t}=\left\{(x, t) \in Q_{T}: u(x, t) \neq v(x, t), x \in D_{\lambda}\right\}, \\
& D_{4 t}=\left(\Omega \backslash D_{\lambda}\right) \cap\{x \in \Omega: u(x, t) \neq v(x, t)\},
\end{aligned}
$$


then

$$
\begin{aligned}
& \int_{0}^{t} \int_{D_{1 t}} a(x)|u|^{\alpha(x)}\left(|\nabla u|^{p(x)-2} \nabla u-|\nabla v|^{p(x)-2} \nabla v\right) \nabla \chi_{\lambda}(u-v) g_{\eta}^{\prime}(u-v) d x d t \\
& \quad=0
\end{aligned}
$$

and

$$
\begin{aligned}
& \int_{0}^{t} \int_{D_{1 t}} a(x)\left(|u|^{\alpha(x)}-|v|^{\alpha(x)}\right)|\nabla v|^{p(x)-2} \nabla v \nabla \chi_{\lambda}(u-v) g_{\eta}^{\prime}(u-v) d x d t \\
& \quad=0
\end{aligned}
$$

In the fourth place, since

$$
\int_{0}^{t} \int_{D_{\lambda}} a(x)|\nabla u|^{p(x)} d x d t \leq c, \quad \int_{0}^{t} \int_{D_{\lambda}} a(x)|\nabla v|^{p(x)} d x d t \leq c,
$$

by (4.3),

$$
\begin{aligned}
& \mid \int_{0}^{t} \int_{D_{2 t}} a(x)\left(|\nabla u|^{p(x)-2} \nabla u-|\nabla v|^{p(x)-2}\right) \nabla v \nabla \chi_{\lambda} g_{\eta}(u-v) d x d t \mid \\
&=\left|\int_{0}^{t} \int_{D_{2 t} \cap D_{3 t}} a(x)\left(|\nabla u|^{p-2} \nabla u-|\nabla v|^{p-2} \nabla v\right) \nabla \chi_{\lambda} g_{\eta}(u-v) d x d t\right| \\
&+\left|\int_{0}^{t} \int_{D_{4 t}} a(x)\left(|\nabla u|^{p-2} \nabla u-|\nabla v|^{p-2} \nabla v\right) \nabla \chi_{\lambda} g_{\eta}(u-v) d x d t\right| \\
&=\left|\int_{0}^{t} \int_{D_{4 t}} a(x)\left(|\nabla u|^{p-2} \nabla u-|\nabla v|^{p-2} \nabla v\right) \nabla \chi_{\lambda} g_{\eta}(u-v) d x d t\right| \\
& \leq \frac{1}{\lambda} \int_{\Omega \backslash D_{\lambda}} a(x)\left(|\nabla u|^{p(x)-2} \nabla u-|\nabla v|^{p(x)-2} \nabla v\right)|\nabla \chi| d x \\
& \leq \int_{0}^{t}\left[\left(\int_{\Omega \backslash D_{\lambda}} a(x)\left(|\nabla u|^{p(x)}+|\nabla v|^{p(x)}\right) d x\right)^{\frac{1}{q^{+}}}\right. \\
& \quad \cdot \frac{1}{\lambda}\left(\int_{\Omega \backslash D_{\lambda}} a(x)|\nabla \chi|^{p(x)} d x\right)^{\frac{1}{p^{+}}} d t \\
& \quad \rightarrow 0
\end{aligned}
$$

as $\lambda \rightarrow 0$. Here, we have used the fact

$$
\int_{\Omega \backslash D_{\lambda}} a(x)|\nabla \chi|^{p(x)} d x \leq \int_{\Omega \backslash \Omega_{\lambda}} a(x)|\nabla \chi|^{p(x)} d x
$$

At last, for the nonlinear damping term satisfying (3.1), we can deal with it as in Theorem 3.3, we omit the details here.

Letting $\eta \rightarrow 0$ in (4.4), let $\lambda \rightarrow 0$. The Gronwall inequality yields the conclusion. 
Proof of Theorem 1.5 Only if we choose $\chi(x)=a(x)$, then

$$
\frac{1}{\lambda}\left(\int_{\Omega \backslash D_{\lambda}} a(x)|\nabla \chi|^{p(x)} d x\right)^{\frac{1}{p^{+}}} \leq\left(\lambda^{1-p^{+}} \int_{\Omega \backslash \Omega_{\lambda}}|\nabla \chi|^{p(x)} d x\right)^{\frac{1}{p^{+}}} \leq c,
$$

and the conclusion follows clearly.

\section{Acknowledgements}

The author would like to thank Dr. Zhan for his kind help!

Funding

Not applicable.

Availability of data and materials

Not applicable.

\section{Competing interests}

The author declares that they have no competing interests.

\section{Authors' contributions}

The author read and approved the final manuscript.

\section{Publisher's Note}

Springer Nature remains neutral with regard to jurisdictional claims in published maps and institutional affiliations.

Received: 22 September 2020 Accepted: 31 January 2021 Published online: 18 February 2021

\section{References}

1. Antontsev, S., Shmarev, S.: Parabolic equations with double variable nonlinearities. Math. Comput. Simul. 81, 2018-2032 (2011)

2. Antontsev, S.N., Shmarev, S.I.: Parabolic equations with double variable nonlinearities. Math. Comput. Simul. 81, 2018-2032 (2011)

3. Antontsev, S.N., Shmarev, S.l.: Evolutions PDES with Nonstandard Growth Conditions. Existence, Uniqueness, Localization, Blow-up, Atlantis Studies in Differential Equations, vol. 4. Series Editor: Michel Chipot. Atlantis Press, Paris (2015)

4. Benedikt, J., Bobkov, V.E., Girg, P., Kotrla, L., Takac, P.: Nonuniqueness of solutions of initial-value problems for parabolic p-Laplacian. Electron. J. Differ. Equ. 2015, 38 (2015)

5. Bertsch, M., Dal Passo, R., Ughi, M.: Discontinuous viscosity solutions of a degenerate parabolic equation. Transl. Am. Math. Soc. 320,779-798 (1990)

6. Fan, X.L., Zhao, D.: On the spaces $L^{p(x)}(\Omega)$ and $W^{m, p(x)}$. J. Math. Anal. Appl. 263, 424-446 (2001)

7. Gu, L:: Second Order Parabolic Partial Differential Equations. The Publishing Company of Xiamen University, Xiamen (2002) (in Chinese)

8. Guo, B., Gao, W.: Extinction and asymptotic behavior of solutions for nonlinear parabolic equations with variable exponent of nonlinearity. Acta Math. Sci. 32B(3), 1053-1062 (2012)

9. Ho, K., Sim, I.: On degenerate $p(x)$-Laplacian equations involving critical growth with two parameters. Nonlinear Anal. $132,95-114(2016)$

10. Karlsen, K.H., Ohlberger, M.: A note on the uniqueness of entropy solutions of nonlinear degenerate parabolic equations. J. Math. Anal. Appl. 275, 439-458 (2002)

11. Kobayasi, V., Ohwa, H.: Uniqueness and existence for anisotropic degenerate parabolic equations with boundary conditions on a bounded rectangle. J. Differ. Equ. 252, 137-167 (2012)

12. Kovácik, O., Rákosník, J.: On spaces $L^{p(x)}$ and $W^{k, p(x)}$. Czechoslov. Math. J. 41, 592-618 (1991)

13. Li, Y., Wang, Q.: Homogeneous Dirichlet problems for quasilinear anisotropic degenerate parabolic-hyperbolic equations. J. Differ. Equ. 252, 4719-4741 (2012)

14. Lian, S., Gao, W., Yuan, H., Cao, C.: Existence of solutions to an initial Dirichlet problem of evolutional $p(x)$-Laplace equations. Ann. Inst. Henri Poincaré, Anal. Non Linéaire 29, 377-399 (2012)

15. Lions, P.L., Perthame, B., Tadmor, E.: A kinetic formation of multidimensional conservation laws and related equations. J. Am. Math. Soc. 7, 169-191 (1994)

16. Ruzicka, M.: Electrorheological Fluids, Modeling and Mathematical Theory. Lecture Notes in Math., vol. 1748. Springer, Berlin (2000)

17. Simon, J.: Compact sets in the space $L^{P}(0, T ; B)$. Ann. Mat. Pura Appl. 146(1), 65-96 (1986)

18. Taylor, M.E.: Partial Differential Equations III. Springer, Berlin (1999)

19. Tersenov Alkis, S., Tersenov Aris, S.: Existence of Lipschitz continuous solutions to the Cauchy-Dirichlet problem for anisotropic parabolic equations. J. Funct. Anal. 272, 3965-3986 (2017)

20. Yin, J., Wang, C.: Properties of the boundary flux of a singular diffusion process. Chin. Ann. Math. 25B(2), 175-182 (2004)

21. Yin, J., Wang, C.: Evolutionary weighted $p$-Laplacian with boundary degeneracy. J. Differ. Equ. 237, 421-445 (2007) 
22. Zhan, H.: The boundary value condition of an evolutionary $p(x)$-Laplacian equation. Bound. Value Probl. 2015, 112 (2015)

23. Zhan, H.: Solutions to polytropic filtration equations with a convection term. Electron. J. Differ. Equ. 2017, 207 (2017)

24. Zhan, H.: Infiltration equation with degeneracy on the boundary. Acta Appl. Math. 25B(2), 147-161 (2018)

25. Zhan, H.: The uniqueness of the solution to the diffusion equation with a damping term. Appl. Anal. 98(7), 1333-1346 (2019)

26. Zhan, H.: On anisotropic parabolic equations with a nonlinear convection term depending on the spatial variable. Adv. Differ. Equ. 2019, 27 (2019)

27. Zhan, H., Feng, Z: Solutions of evolutionary $p(x)$-Laplacian equation based on the weighted variable exponent space. Z. Angew. Math. Phys. 68, 134 (2017)

28. Zhan, H., Feng, Z.: Degenerate non-Newtonian fluid equation on the half space. Dyn. Partial Differ. Equ. 15(3), 215-233 (2018)

29. Zhan, H., Feng, Z.: The well-posedness problem of an anisotropic parabolic equation. J. Differ. Equ. 268, 389-413 (2020)

30. Zhan, H., Feng, Z.: Stability of anisotropic parabolic equations without boundary conditions. Electron. J. Differ. Equ. 2020, 74 (2020)

31. Zhang, Q., Shi, P.: Global solutions and self-similar solutions of semilinear parabolic equations with nonlinear gradient terms. Nonlinear Anal. TMA 72, 2744-2752 (2010)

32. Zhikov, V.V.: On the density of smooth functions in Sobolev-Orlicz spaces. Otdel. Mat. Inst. Steklov. (POMI) 310, 67-81 (2004). Translation in J. Math. Sci. NY 132, 285-294 (2006)

33. Zhou, W., Cai, S.: The continuity of the viscosity of the Cauchy problem of a degenerate parabolic equation not in divergence form. J. Jilin Univ. Nat. Sci. 42, 341-345 (2004)

\section{Submit your manuscript to a SpringerOpen ${ }^{\circ}$ journal and benefit from:}

- Convenient online submission

- Rigorous peer review

- Open access: articles freely available online

- High visibility within the field

- Retaining the copyright to your article

Submit your next manuscript at $\boldsymbol{\nabla}$ springeropen.com 